\title{
Analysis of Risk Factors for Incisional Hernias and its Management
}

\author{
Paudel $\mathrm{SR}^{1^{*}}$, Neupane $B R^{2}$, Gurung $N V^{3}$, Acharya $A^{4}$, Chapagain $A^{5}$, Shrestha $S^{5}$, Gurung $A^{5}$, Shrestha $D^{5}$ \\ ${ }^{1}$ Consultant Surgeon, Fewa City Hospital \& Western Regional Hospital, Pokhara, Nepal \\ ${ }^{2}$ Sr. consultant, Fewa City Hospital \& Gandaki Medical College \& Teaching Hospital, Pokhara,Nepal \\ ${ }^{3}$ Chief consultant Surgeon, ${ }^{4}$ Consultant Surgeon, Western Regional Hospital, Pokhara, Nepal \\ ${ }^{5}$ Surgeons, Western Regional Hospital, Pokhara, Nepal
}

\author{
Keywords \\ Incisional hernia, Lower abdominal \\ midline incision, Mesh repair. \\ Corresponding author \\ *Dr. Suresh Raj Paudel \\ Consultant Surgeon \\ Western Regional Hospital \\ Pokhara, Nepal \\ Email: poudelsuresh6@gmail.com
}

\begin{abstract}
Introduction: Incisional hernia is a common problem after abdominal surgery. Patients present with pain, swelling and intestinal obstruction. It may be repaired by either anatomical suturing or mesh repair.
\end{abstract}

Methods: It is a prospective observational study conducted in Western Regional Hospital and Fewa City Hospital, Pokhara from 2013 to 2016. A total of consecutive 100 patients admitted in these hospitals during the study period were included.

Results: Incisional hernia is more common in females ( $\mathrm{M}: \mathrm{F}=1: 3.8$ ), and in 30 - 50 years age group (60\%). Major risk factors were wound infection (30\%), overweight (25\%), and postoperative cough (10\%). It is found to be more associated with gynecological (65\%), than gastrointestinal operations, and more so with lower abdominal midline incision (65\%). It is found to occur mostly within one year $(60 \%)$ of primary surgery than later. Even $24 \%$ of the patients had first symptom within six months. Mesh repair (92\%) was the preferred standard surgical treatment for incisional hernia.

Conclusion: Overweight females of age range between 30 - 50 years with history of gynecological operations by lower abdominal midline incision are more prone to develop incisional hernia. This incidence increases when there is wound infection. Mesh repair is the choice of operation for incisional hernia.

\section{INTRODUCTION}

Incisional hernia is a common presentation in general surgery. It account for 15 to $20 \%$ of all abdominal hernias. They develop in two to 11 percent of patients undergoing laparotomy. The figure rises to $26 \%$ in those who develop wound infection ${ }^{1}$. In 80 to $95 \%$ of the patients they develop within six months to three years after initial surgery $^{2}$. Hernias that develop within three years of operation are more likely to be troublesome and larger in size than those that develop later ${ }^{3}$.
Approximately 200,000 incisional hernia repairs are performed each year in the United States and it is estimated that another 200,000 incisional hernias may be going unrecognized or untreated. Incisional hernias occur mostly with midline and transverse incision. It also occurs with paramedian, subcostal, McBurney, Pfannenstiel, flank incisions, and laparoscopic port sites. An incisional hernia occurs early in the healing process due to biochemical failure of the acute fascial wound when wound tensile strength is very low or absent ${ }^{4}$. 
During this time, the wound strength depends entirely on suture integrity. So when patients start returning to the increased levels of activity and place loads across their acute wounds, the wound gives away. However, the hernia may not be obvious for days or even years.

According to Jack Abrahmson, many factors singly or in combination may cause failure of the wound to heal. The factors responsible for the failure include obesity, wound infection, closure of fascia with catgut, drainage tube through the index incision, senility, early wound dehiscence, immunosuppressant therapy, anemia, diabetes mellitus, malnutrition, jaundice and azotemia. Suture length and technique also play a role. Repair of abdominal incisional hernia is difficult with recurrence. Recurrence rates of up to 33\% after first repair and 58\% after second repair have been reported ${ }^{5}$ with anatomical repair. Meshes have reduced the recurrence rate to 1 $-10 \%$.

The aim of this study is to determine the common risk factors for incisional hernias such as age and sex of the patient, predisposing factors like overweight, previous wound infection, chronic urinary retention, diabetes mellitus, postoperative cough, COPD, type of previous surgery, type of previous incision, duration from previous surgery. This study will also determine the common surgery for incisional hernia.

\section{METHODS}

It was a prospective, observational study conducted in Western Regional Hospital and Fewa City Hospital, Pokhara from 2013 to 2016. A total of one hundred consecutive patients of incisional hernia admitted in those hospitals during the study period were included in the study. After detailed history and a thorough clinical examination, information of the patients was collected in various aspects like age, sex, mode of presentation, previous operation (indication of surgery, incision site, and post-operative wound infection). Patients were also evaluated for other risk factors like obesity, diabetes mellitus and malignant diseases. Routine laboratory investigations of blood, and urine, chest X-ray, and ECG were done. After complete evaluation they were operated either by anatomical closure or with mesh repair. Data were collected on a structured proforma. Statistical Analysis was done using Microsoft Excel software. Result obtained from the study was discussed with reference to current world literature.

\section{RESULTS}

Fig 1: Age and sex distribution of patients with incisional hernia

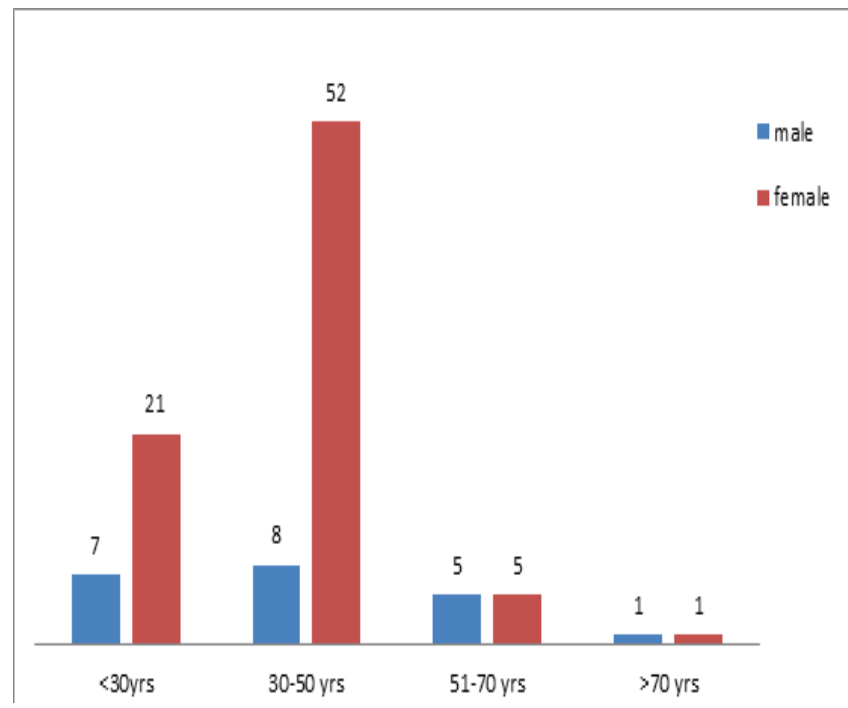

Among one hundred patients enrolled in the study, 21 were males and 79 were females in the ratio of $1: 3.8$. Most of the patients (60\%) were in the age group of 30 50 years. The youngest patient was 21 years old and the oldest was 81 years of age.

Table 1: Predisposing factors in patients with incisional hernias

\begin{tabular}{lc}
\hline \multicolumn{1}{c}{ Predisposing factors } & Number \\
\hline Overweight BMI $>25$ & 25 \\
Previous wound infection & 30 \\
Chronic urinary retention & 3 \\
Diabetes mellitus & 5 \\
Postoperative cough & 10 \\
Chronic obstructive pulmonary disease & 2 \\
No associated factors & 25 \\
\hline
\end{tabular}

In our study, $30 \%$ of the patients had previous surgical wound infection, 25\% were overweight, 10\% had postoperative cough, $5 \%$ were diabetic, $3 \%$ had chronic urinary retention, and $2 \%$ had chronic obstructive pulmonary disease. But $25 \%$ of the patients had developed incisional hernia in spite of having not any associated predisposing factors. 
Fig 2: Type of previous surgery

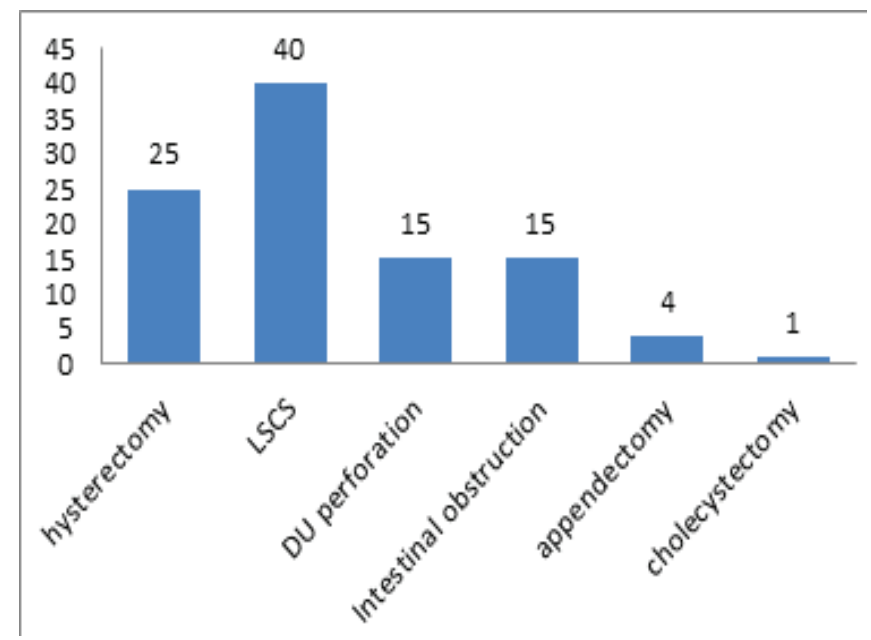

This study showed that $65 \%$ of patients with incisional hernia had undergone gynecological procedures. Among which LSCS was the most common (40\%), followed by abdominal hysterectomy (25\%). Among other surgical procedures followed were laparatomy for duodenal ulcer perforation (15\%), for intestinal obstruction (15\%), appendectomy (4\%) and open cholecystectomy (1\%).

Fig 3: Type of previous incision

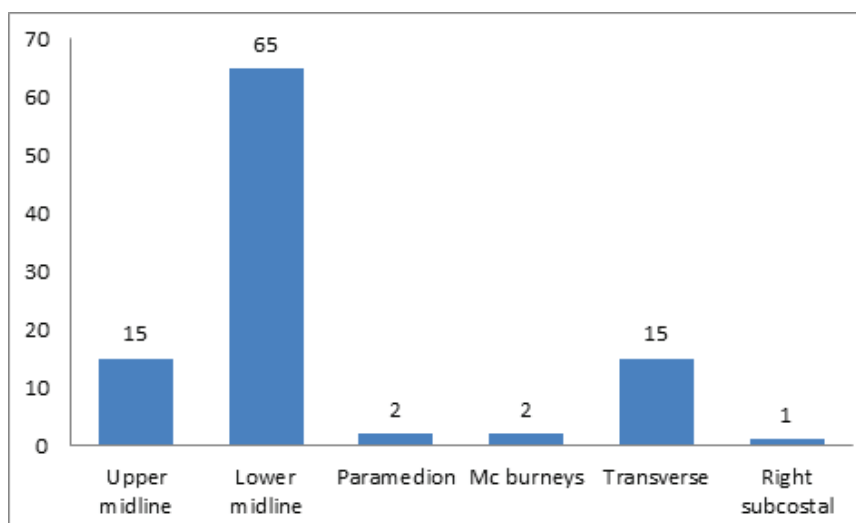

In this study incisional hernias were more common in patients with lower midline incision (65\%), followed by upper midline (15\%), transverse (15\%), McBurney's (2\%), paramedian $(2 \%)$, and right subcostal (1\%).

Fig 4: Duration from previous surgery

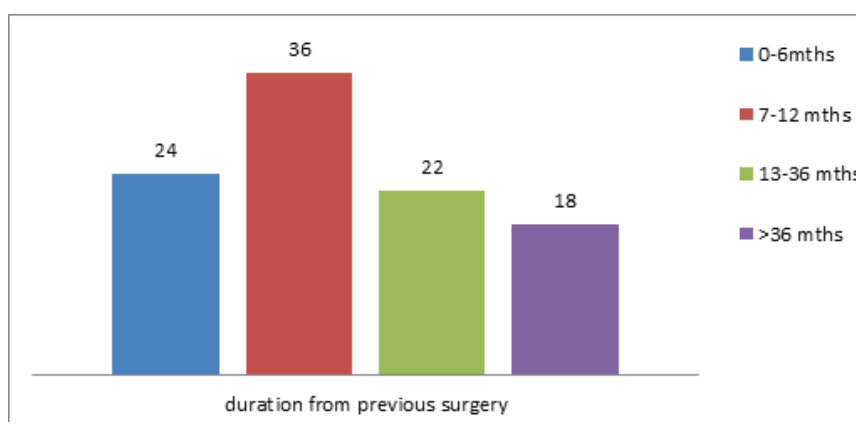

It was found that $24 \%$ of the patients had developed incisional hernia within six months, $36 \%$ of the patients had developed hernia from seven to 12 months, $22 \%$ developed in 13 to 36 months and the rest $18 \%$ after 36 months of primary surgery.

Fig 5: Therapeutic modality

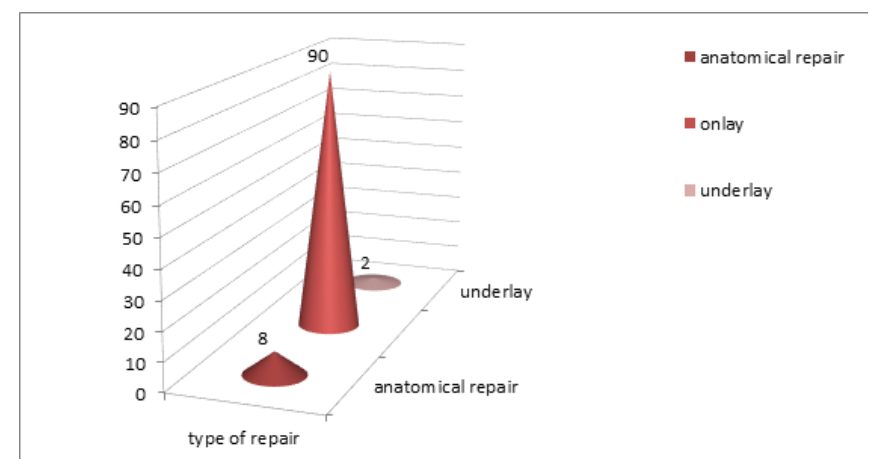

In our study most of the operated incisional hernias $(92 \%)$ were repaired by placing a mesh. Among them $90 \%$ had onlay placement of mesh and two had underlay mesh. Only $80 \%$ of the incisional hernias were repaired anatomically. No recurrence of incisional hernia was found after repair.

\section{DISCUSSION}

Incisional hernia is defined as any abdominal wall gap with or without bulge in the area of a postoperative scar perceptible or palpable by clinical examination or by imaging. Any condition which inhibits natural wound healing leads to the development of an incisional hernia. Those conditions are previous wound infection, wound dehiscence, poor surgical technique, obesity, smoking, diabetes mellitus, immunosuppressive conditions, excessive wound tension, malnutrition, connective tissue disorders, chronic obstructive pulmonary disease, and other co-morbidities. Emergency surgery increases the risk of incisional hernia.

In our study the incidence of incisional hernia was more in 30 to 50 years age group. It was common in females (M $: F=1: 3.8$ ). This is because of laxity of abdominal muscles due to multiple pregnancies and increased incidence of obesity in females. A retrospective study by Hoer J et al found that age around 45 years, female gender, BMI $>25$, and previous laparotomies played an important role in the development of incisional hernia ${ }^{6}$. Similarly another retrospective study by Bhat $\mathrm{M}$ et al showed that incisional hernia was common in females in the fifth decade and gynecological operation with lower midline incision was 
the most common predisposing factors for development of incisional hernia ${ }^{7}$. These studies were comparable with our study.

In our study major predisposing factors were previous wound infection (30\%), overweight (25\%), postoperative cough (10\%), diabetes mellitus (5\%), COPD (2\%) and chronic urinary retention (3\%). These results were comparable with those of Bose et $a l^{8}$ in which the predisposing factors were wound infection (53.63\%), obesity (30\%), COPD (20.90\%) and urethral stricture (9\%). Similarly a prospective study by Agbakwuru EA et al found that midline incisions, wound sepsis, and overweight are the major risk factors ${ }^{4}$. Colombo et al also reported that the incisional hernia occurring within three years after midline abdominal incision for gynecologic diseases was associated with obesity and anesthesiological risk factors ${ }^{9}$.

Incisional hernia in diabetic patients occurs due to altered regulation of collagen metabolism at the level of the fascial scar. When diabetes was optimally controlled, wound strength and extensibility were similar in diabetic and non-diabetic ${ }^{10}$. So the association between incisional hernia and diabetes might be explained by the suboptimal glycemic control often found in elderly diabetic subjects and not by the disease per se.

Wound dehiscence is defined as failure of the incision to heal and maintain a normal abdominal wall anatomy. It presents with serosanguinous discharge from the wound in the first week of surgery. The severity ranges from superficial breakdown of the skin to a complete failure and an exposure of the viscera. Incisional hernia is chronic wound failure ${ }^{11}$. A study carried out in Egypt concluded that incisional hernia is a complication of wound healing after surgery which can be prevented by good care and precaution $^{12}$.

In our study incisional hernia was common in patients with previous lower abdominal midline incision like in LSCS (40\%), hysterectomy (25\%), intestinal obstruction (15\%). Lower abdominal incisions have a higher incidence of development of incisional hernia due to absence of posterior rectus sheath below arcuate line, increased intra-abdominal hydrostatic pressure in erect position and laxity of abdominal muscles in females. This study was comparable with the study by Parekh JN, Shah DB, Thakore et $a l^{13}$. A randomized trial comparing vertical and transverse incisions for abdominal aortic aneurysm repair found that incisional hernia was more likely to occur with vertical laparotomy (20 out of 22 patients with vertical incisions versus six out of 15 patients with transverse incision laparotomy $)^{14}$. Incisional hernias have been described following paramedian, subcostal, McBurney, Pfannenstiel, and flank incisions. Incisional hernias are also reported in laparoscopic port sites due to weakness in the fascia.

Our study result of $60 \%$ of incisional hernias occurring within one year of primary surgery is comparable with Akmans series with $65 \%$ of hernia occurring within a year. In our study, $24 \%$ of cases had developed incisional hernia within six months, $36 \%$ cases had it in seven to 12 months, $22 \%$ from 13 to 36 months and 18\% after 36 months of previous surgery.

Most of the incisional hernias should be repaired in fear of more sinister complications. Upper abdominal hernias, hernias less than one $\mathrm{cm}$ in diameter, and hernias larger than seven to eight $\mathrm{cm}$ are less likely to incarcerate. Surgery should be considered when it is symptomatic, has potential for incarceration, and when the hernia size is sufficient enough to hamper activities of daily living.

There are two basic types of repair: anatomical repair and mesh repair. Mesh repairs are preferable for incisional hernias. In a retrospective study from Europe, the incidence of recurrence of incisional hernias after simple sutured repair was over $60 \%$, and the use of mesh decreased the recurrence rate to approximately $17 \%{ }^{15}$. Continued smoking and occupational straining may be the risk factors for the recurrence of mesh repair in ventral abdominal hernia ${ }^{16}$. Mesh repair is important for incisional hernia with a diameter greater than four $\mathrm{cm}$ as the risk of recurrence is related to the tension placed on the repair in large hernias. Suture repairs have certain advantages like nidus for infection is less; less chance of adhesion to bowel and ability to close some very small hernias without tension.

Mesh repairs minimize the amount of tension that must be put on the abdominal wall in order to cover the hernia. The mesh can be placed above the fascia (onlay), below the fascia (sublay), or simply bridge the gap of the defect by suturing the mesh to the fascial edges and underlay. Laparoscopic repair of incisional hernia is a relatively new procedure. Laparoscopic repair most closely mirrors the sublay technique of open repair. A large piece of mesh with a minimum of four to five $\mathrm{cm}$ overlap over the hernia is secured with transfascial sutures and intra-abdominally placed tacks. 


\section{SUMMARY}

Incisional hernia is common in females of age range 30 to 50 years with history of gynecological operation by lower abdominal midline incision like in LSCS and abdominal hysterectomy. Previous wound infection, overweight and post operative cough are important predisposing factors. It usually occurs within the first year of previous surgery. Mesh repair is the preferred choice of repair.

\section{REFERENCES}

1. Bucknall TE, Cox PJ, Ellis H. Burst abdomen and incisional hernia: A prospective study of 1129 major laparotomies. BMJ. 1982; 284: 931-3.

2. Pilaay Y, Naidoo NM, Madiha TE. Incisional hernia in a single surgical unit. East and Central African Journal of Surgery. 2007 April 1; 12(1): 42-46.

3. Mudge M, Hughes LE. Incisional hernia: A 10 year prospective study of incidence and attitudes. $\mathrm{Br} J$ Surg. 1985: 72: 70-71.

4. Agbakwuru EA, Olabanji JK, Alatise OI, Okwerekwu RO, Esimai OA. Incisional hernia in women: Predisposing factors and management where mesh is not readily available. Libyan J Med. 2009; 4(2): 84-89.

5. Luijendijik RW, Hop WCJ, Tol MP. A comparison of suture repair with mesh repair for incisional hernia. N Engl J Med. 2000; 343: 392-398.

6. Höer J, Lawong G, Klinge U, Schumpelick V. Factors influencing the development of incisional hernia. A retrospective study of 2,983 laparotomy patients over a period of 10 years. Chirurg. 2002; 73(5): 47480.

7. Bhat M, Somasundaram S. Preperitoneal mesh repair of incisional hernias: A seven-year retrospective study. Indian Journal of Surgery. 2007 May 1.

8. Bose SM, Lal Roshan, Kalra Manju, Wig JD, Khanna SK. Ventral hernia - A review of 175 cases. Indian Journal of Surgery. 1999; 61(3):180-84.

9. Colombo M, Maggioni A, Parma G, Scalambrino S, Milani R. A randomized comparison of continuous versus interrupted mass closure of midline incisions in patients with gynecologic cancer. Obstet Gynecol. 1997; 89: 684-9.

10. Yue DK, McLennan S, Marsh M, May YW, Spaliviero J, Delbridge L et al. Effects of experimental diabetes, uremia and malnutrition on wound healing. Diabetes. 1987; 36: 295-9.

11. Shoo YW, Andrew NK. Abdominal wound dehiscence and incisional hernia. Online. In print Practice Journal of Surgery. 2002 may 1; 20(5); 100-103.

12. Ehab E and Alaa A. Huge incisional hernia: A case report. Cases Journal. 2008; 1: 202.

13. ParekhJN, Shah DB, Thakore AB. Incisional hernia- A study of 76 cases. Indian Journal Of Surgery. 1988; 50: 49-53.

14. Fassiadis N, Roidl M, Hennig M et al. Randomized clinical trial of vertical or transverse laparotomy for abdominal aortic aneurysm repair. Br J Surg. 2005; 92: 1208.

15. Burger JW, Luijendijk RW, Hop WC et al. Long-term follow-up of a randomized controlled trial of suture versus mesh repair of incisional hernia. Ann Surg. 2004; 240: 578.

16. John L, Clark MD. Ventral Incisional Hernia Recurrence. Journal of Surgical Research. 2001 July; 99(1): 33-39. 